\title{
Resección Quirúrgica de Tumor Maligno Localizado en el Tracto Biliar Distal: Informe de un caso clínico
}

\section{Surgical Resection of Malignant Tumor Located in the Distal Biliary Tract: Report of a clinical case}

${\text { Valeria Grijalva Ozaetta }{ }^{1, *}, \text { Fernando Orejuela Cabrera }}^{2}$

INFORMACIÓN DEL

ARTÍCULO

Fecha de recepción: 22 de octubre de 2019.

Fecha de aceptación: 28 de enero de 2020.

1 Médico Residente Hospital Luis Vernaza - Universidad Espíritu Santo Guayaquil, Ecuador, Dirección Loja 700 y Escobedo, Código Postal 090306.

${ }^{2}$ Médico Residente Hospital Teodoro Maldonado Carbo - Universidad Espíritu Santo - Guayaquil, Ecuador, Dirección Av. 25 de julio y Av. Ernesto Albán, Código Postal 090510.

* Autor de correspondencia: Valeria Grijalva Ozaetta, 1Médico Residente Hospital Luis Vernaza Universidad Espíritu Santo - Guayaquil, Ecuador, Dirección Loja 700 y Escobedo, Código Postal 090306.

E-mail: valeria.grijalva.vg@gmail.com

ENLACE DOI: http://dx.doi.org/10.31095/investigatio 2020.13.4

\begin{abstract}
Resumen
El colangiocarcinoma distal es un tumor maligno altamente letal. El principal factor de riesgo carcinogénico es la inflamación crónica de las vías biliares. Actualmente, la cirugía de Whipple continúa siendo la única opción de tratamiento con intento curativo. Se plantea identificar pacientes con este tipo de tumor y elegir el método de cirugía idóneo para maximizar los beneficios del paciente. Se reportó el caso de un paciente masculino de 62 años de edad ex fumador y alcohólico hace 20 años. Presentó dolor abdominal intermitente, dispepsia y pérdida de apetito. Las pruebas de imagen evidencian vesícula biliar de gran tamaño con dilatación del tercio superior de la vía biliar con estrechez en segmento distal. Se eligió cirugía de Whipple, por laparotomía. El paciente presentó complicaciones hemodinámicas posquirúrgicas por lo que se concluye que la cirugía abierta genera estrés traumático y debería ser manejada por personal médico y hospitales de alto nivel.
\end{abstract}

\section{Palabras Clave:}

Cáncer periampular; procedimiento Whipple; pancreatoduodenectomia abierta.

Clasificación JEL: I12.

\begin{abstract}
Distal cholangiocarcinoma is a highly lethal malignant tumor. The main carcinogenic risk factor is chronic inflammation of the bile ducts. Currently, Whipple surgery remains the only treatment option with curative intent. It is proposed to identify patients with this type of tumor and choose the ideal surgery method to maximize patient benefits. The case of a 62 -year-old male patient, former smoker and alcoholic, has been reported for 20 years. I have intermittent abdominal pain, dyspepsia and loss of appetite. Imaging tests show large gallbladder with dilation of the upper third of the bile duct with narrowness in the distal segment. Whipple surgery was chosen due to laparotomy. The patient presented postoperative hemodynamic complications, so it is concluded that open surgery generates traumatic stress and should be managed by medical personnel and high-level hospitals.
\end{abstract}

Keywords:

Periampular cancer; Whipple procedure; open pancreatoduodenectomy. JEL Classification: I12.

41

INVESTIGATIO No. 13, marzo 2020, pp. 41-48,

ISSN: 1390 - 6399・ISSN-e: 2602 - 8336 


\section{Introducción}

Los colangiocarcinomas (CCA) son un grupo heterogéneo de tumores malignos de tipo epitelial y de crecimiento lento originados en el árbol biliar; representando la segunda neoplasia maligna hepática más común después del carcinoma hepatocelular (CHC) (Rizvi et al., 2017). Se clasifica según su localización anatómica en tres regiones: intrahepáticos, extrahepáticos proximales y extrahepáticos distales. El Colangiocarcinoma que afecta al conducto biliar común distal (colangiocarcinoma distal) o DCC según sus siglas en inglés, es una neoplasia periampular, comprende aproximadamente el $30 \%$ de todos los colangiocarcinomas; poco común considerada una enfermedad altamente letal y que a menudo, es difícil de distinguir comparado con el adenocarcinoma pancreático (PDA). El único tratamiento óptimo para el colangiocarcinoma distal es la resección quirúrgica, como resultado de la insensibilidad del colangiocarcinoma a la radiación y la quimioterapia; sin embargo, el pronóstico y la tasa de curación del DCC han mejorado con respecto a la PDA en los últimos años (Cardinale et al., 2010).

Más del 90\% de estos tumores que se originan en el epitelio de vías biliares corresponden adenocarcinomas. Bartolome (Ridruejo y Jimenez, 2006). Además, pueden presentarse otras variantes histopatológicas como los carcinomas espinocelulares y mucoepidermoideo, tumores de tipo leimiosarcoma, rabdomiosarcoma, cistoadenocarcinoma, carcidenoide y tumores de células granulares (Burgos, 2008).

La inflamación crónica de las vías biliares se considera el principal factor de riesgo carcinogénico y se asocia a la formación de citoquinas por las células inflamatorias y los colangiocitos. Siendo la más frecuente la colangitis esclerosante primaria. A nivel mundial, la incidencia de colangiocarcinoma intrahepático puede estar aumentando, mientras que los colangiocarcinomas extrahepáticos perihilares y distales están disminuyendo. Aunque es más común en Asia, su incidencia en Europa y América del Norte ha aumentado significativamente en las últimas décadas probablemente debido a diferencias genéticas y variaciones geográficas en los factores de riesgo (Labib et al., 2019)

No existe ninguna prueba de laboratorio ni marcadores tumorales específicos para este tumor. La mayoría de los pacientes tienen esta enfermedad en estado avanzado y el diagnóstico de colangiocarcinoma en una etapa temprana sigue siendo un desafío debido a su carácter clínico 'silencioso' (la mayoría de los pacientes con enfermedad en etapa temprana son asintomáticos), la ubicación anatómica de difícil acceso y la naturaleza paucicelular altamente desmoplástica, que limita la sensibilidad del diagnóstico citológico y patológico. No obstante, las técnicas citológicas avanzadas, como la hibridación fluorescente in situ (FISH) y el análisis mutacional, han surgido como modalidades diagnósticas esenciales. (Rizvi et al., 2017) 
Actualmente las pruebas de imagen continúan siendo el pilar de apoyo para el diagnóstico de esta patología. La tomografía computarizada permite llegar al diagnóstico y es especialmente necesaria para la estatificación de las lesiones avanzadas y la resonancia magnética es útil para establecer el grado de invasión a nivel del ligamento hepatoduodenal, del marco portal y para el estudio ganglionar (Ridruejo y Jimenez, 2006).

Los pacientes con imagenología y endoscopia que sugieran una enfermedad locorregional, y con un estado de rendimiento adecuado, deben someterse a una exploración para una posible pancreaticoduodenectomía; una vez resecados, los cirujanos y patólogos deben trabajar juntos para marcar los márgenes críticos de la pieza quirúrgica por lo que se ha demostrado sistemáticamente que los márgenes de resección al ser positivo y las metástasis de los ganglios linfáticos conducen a un mal pronóstico después de la pancreaticoduodenectomía (Mumtaz et al., 2007).

La pancreatoduodenectomía o procedimiento de Whipple, continúa siendo hoy en día la única opción de tratamiento con intento curativo para la enfermedad tumoral periampular, que comprende el adenocarcinoma pancreático, colangiocarcinoma distal, adenocarcinoma ampular y adenocarcinoma duodenal. La evolución de este procedimiento quirúrgico inicio con Whipple en 1935, cuando realizó la resección en 2 tiempos. En 1994, continua con Gagner reportando la primera pancreatoduodenectomía realizada por vía laparoscópica, la cual en un principio tuvo una mala aceptación debido a la complejidad, múltiples anastomosis y prolongado tiempo quirúrgico (ChapaAzuela et al., 2017).

A partir del 2003, la cirugía robótica se utilizó por primera vez para la resección pancreática y se está utilizando cada vez más. De acuerdo con un metanálisis del 2016 se reporta nueve estudios comparativos con 246 procedimientos robóticos versus 391 laparoscópicos. Los resultados a corto plazo en términos de morbilidad postoperatoria, hospitalización y recuperación fueron similares (Buanes y Edwin, 2018).

La asistencia robotizada ofrece varias ventajas como complemento de la cirugía laparoscópica, incluido el aumento de destreza, visualización tridimensional, una disección más precisa y una construcción más segura de las anastomosis por lo que actualmente se requiere adiestramiento del médico cirujano en el área robótica (Lalli et al., 2018). Debido a la importancia y lo compleja que suele ser el manejo quirúrgico de esta patología, el autor se ve en la necesidad de brindar información que sirva de apoyo para futuros avances en la técnica quirúrgica y la disposición de herramientas tecnológicas utilizadas en el área de la salud. El propósito de este estudio es identificar pacientes con este tipo de tumor y elegir el método de cirugía idóneo para maximizar los beneficios del paciente.

\section{Resultados}

De acuerdo a los hallazgos encontrados en las pruebas de imagen se decidió realizar la cirugía de Whipple por laparotomía. 
Las probabilidades de recuperación del paciente eran estrechamente positivas si se sometía a la Cirugía de Wipple con la finalidad de extraer el tumor, aumentando, en el mejor de los casos, su calidad de vida durante un par de años más; sin embargo, el manejo clínico y quirúrgico del paciente $\mathrm{y}$, su respuesta orgánica ante este procedimiento no fue favorable, por lo que hubo alteración hemodinámica, debido a un síndrome de respuesta inflamatoria sistémica severo con una puntación de APACHE II 24 acompañado de distrés respiratorio, falla renal aguda y acidosis metabólica. De acuerdo a tales complicaciones quirúrgicas el paciente falleció.

Presentación de caso: Se reporta caso clínico de un paciente masculino de 62 años de edad con antecedente patológico personal de fumador y alcoholismo de aproximadamente 20 años de evolución, presenta cuadro clínico de 3 meses de evolución caracterizado por dolor abdominal tipo cólico, intermitente y de localización en hipocondrio derecho que se irradia hacia hipocondrio izquierdo acompañado de dispepsia y pérdida de apetito motivo por el cual es ingresado al hospital Luis Vernaza el día 18 de enero del 2015. Tres días antes de ingresar al hospital, el paciente ingiere buscapina cada 8 horas $y$ productos naturales para aliviar el dolor; sin embargo, presenta ictericia, acolia, coluria más reagudización del cuadro clínico anteriormente descrito el día 18. A su ingreso se encontró con tinte ictérico generalizado de piel y mucosas, los estudios de Laboratorio reportaron perfil hepático-pancreático y marcador tumoral con valores elevados de acuerdo a la Tabla 1.
Tabla 1.

Valores de laboratorio del paciente al ingreso

\begin{tabular}{ll}
\hline Párametro & Valor \\
\hline Hemoglobina & $13,3 \mathrm{~g} / \mathrm{dl}$ \\
Hematocrito & $40 \%$ \\
Urea & $24 \mathrm{mg} / \mathrm{dl}$ \\
Creatinina & $1,11 \mathrm{mg} / \mathrm{dl}$ \\
TGO & $109 \mathrm{u} / \mathrm{l}$ \\
TGP & $276 \mathrm{u} / 1$ \\
GGT & $1091 \mathrm{u} / 1$ \\
FA & $626 \mathrm{u} / 1$ \\
CA19-9 & $1509 \mathrm{u} / \mathrm{ml}$ \\
Amilasa & $81 \mathrm{u} / 1$ \\
Lipasa & $47 \mathrm{u} / 1$ \\
\hline
\end{tabular}

Los hallazgos encontrados en la tomografía de abdomen superior simple y contrastada se observó el conducto cístico dilatado, con medida de $1,8 \mathrm{~cm}$ de longitud e imagen hipo-densa en su interior además una lesión hipo-densa de bordes lobulados en el lóbulo caudado (segmento I) que mide 3,7 x 3,7 x 3,4 cm, con vesícula aumentada de tamaño, distendida, paredes gruesas y contenido denso. El conducto colédoco prominente presento medida de $7,7 \mathrm{~mm}$ de longitud y un páncreas de morfología conservada, no se observó cambios densitométricos con conducto de tamaño normal (Figura 1).

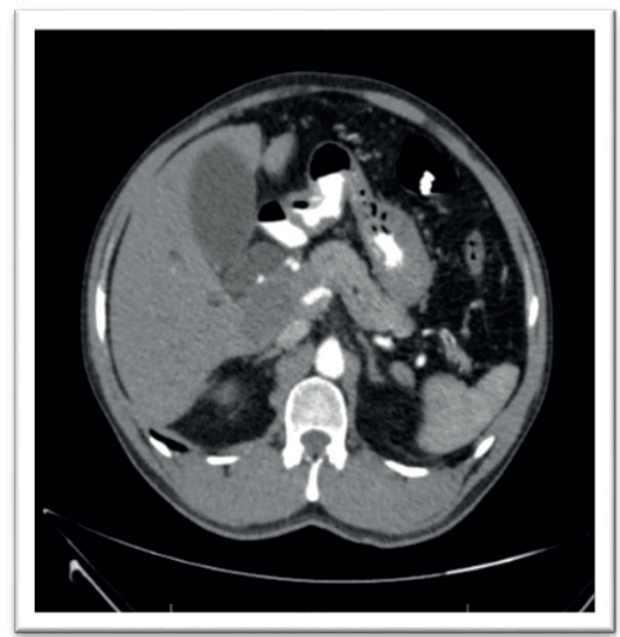

Figura 1. Tomografía de abdomen superior simple y contrastado. 
Se realizó una colangioresonancia, en al cual se observó vesícula biliar de gran tamaño, distendida, de bordes finos, con dilatación del tercio superior de la vía biliar con estrechez en segmento distal o colédoco (Figura 2).

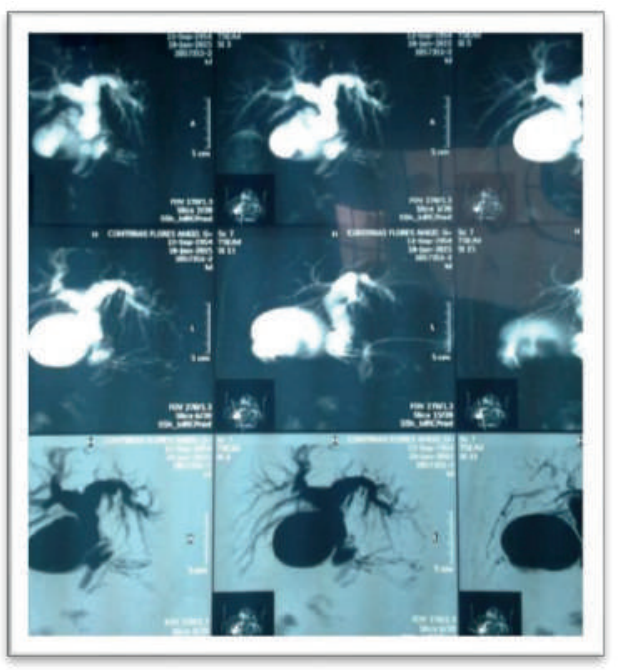

Figura 2. Resonancia Magnética de abdomen.

\section{Resonancia Magnética de abdomen}

Con base en la evaluación diagnóstica y los exámenes complementarios se decidió hacer una laparotomía exploratoria mediante incisión de Chevron, donde se observó masa de consistencia dura con bordes irregulares y de aproximadamente de $7 \mathrm{~cm}$, localizado en región posterior de colédoco distal, por encima de la arteria hepática derecha. También se registró vesícula distendida con paredes gruesas y cabeza pancreática de consistencia fibrótica, por lo que, se llevó a cabo la cirugía de Wipple o duodenopancreatectomia cefálica con colecistectomía y preservación de píloro más exéresis de ganglios periportales.

Se recabaron resultados de la biopsia, en la cual se encontró a nivel del páncreas una neoplasia de estirpe epitelial constituida por células de moderado pleoformismo nuclear y un moderado índice mitótico, dispuestas en forma glandular, compatible con metástasis carcinomatoso. Además, se reportó infiltración de mucosa duodenal comprometiendo la pared del conducto de Wirsung, con presencia de áreas de necrosis y embolias vasculares. Los cortes de la formación nodular presentaron infiltración por dicho tumor. El tumor encontrado durante la cirugía midió $3 \mathrm{~cm}$ de largo y $1 \mathrm{~cm}$ de grueso aproximadamente. Debido a que se encontraba en el tramo de las vías biliares cercano al intestino delgado se concluye que era un Colangiocarcinoma Distal (DCC).

\section{Discusión}

La cirugía de Whipple se realiza con frecuencia en centros de referencia en todo el mundo, representa una de las cirugías abdominales más complejas con alta morbilidad peri-operatoria de 30 a $40 \%$ y una tasa de mortalidad de 1 a $6 \%$ (Kornaropoulos et al., 2017).

La cirugía de Whipple se define como un procedimiento estándar donde el cirujano reseca la cabeza del páncreas, la vesícula biliar, parte del duodeno, una pequeña porción del estómago y los ganglios linfáticos cercanos a la cabeza del páncreas y se realiza la anastomosis del tejido pancreático con los órganos digestivos a fin de que las enzimas pancreáticas digestivas, la bilis y el contenido del estómago se dirigen hacia el intestino delgado durante la digestión (Network, 2019). 
Se ha demostrado en estudio que la cirugía abierta genera gran estrés traumático suprimiendo el sistema inmune por múltiples mecanismos a diferencia de la pancreaticoduodenectomía laparoscópica que ha permitido a los cirujanos ubicar, controlar e incluso diseccionar de manera más precisa la manipulación de pequeños vasos, evitando hemorragias masivas, por lo tanto conduciendo a una menor pérdida de sangre durante la operación y disminuyendo la tasa de transfusión, teniendo en cuenta que la transfusión de sangre intra-operatoria se asocia con recurrencia tumoral y metástasis (Liu et al., 2019).

En el año 2013, en la ciudad de México, se inició el proceso de adiestramiento para la realización de la cirugía de Whipple por vía laparoscópica, que incluyó en su primera etapa solamente la fase de evaluación de la resección, para posteriormente convertir la cirugía abierta y realizar la fase de reconstrucción de manera tradicional; luego se inició el proceso para realizar la segunda fase de reconstrucción por vía laparoscópica, que tuvo como resultado el primer Whipple con preservación de píloro (Chapa et al., 2017).

En el caso del paciente presentado en este estudio, se realizó la cirugía de Wipple abierta con preservación del píloro, donde no se extirpó la porción inferior del estómago; sin embargo, se realizó exéresis de los ganglios periportales y, la cirugía tuvo duración de siete horas con veinticinco minutos. Mediante la intervención se descubrió que el paciente presentaba infiltración en la mucosa duodenal a nivel de la pared del conducto de Wirsung con áreas de necrosis y presencia de vasos trombosados compatible con metástasis carcinomatoso.

Con el desarrollo y avance tecnológico, se realizó el primer procedimiento robótico de Whipple en el 2003, a partir de este acontecimiento algunos autores han evaluado la importancia de la pancreaticoduodenectomía robótica y la pancreaticoduodenectomía abierta en términos de seguridad y eficiencia (Wang et al., 2017).

Se han hecho meta-análisis con recopilación de datos publicados en PubMed, EMBASE, Medline and Cochrane Library referente a las ventajas del uso de cirugía robótica vs cirugía convencional, se menciona que la asistencia robótica reduce la presencia de hemorragia después del procedimiento, se asocia a un tiempo operatorio prolongado con una tasa de resección $\mathrm{R}_{0}$ más alta, breve estancia hospitalaria y baja infección del sitio quirúrgico (Peng et al., 2016).

Para algunos autores, ninguno de los procedimientos como la pancreaticoduodenectomía robótica y la pancreaticoduodenectomía abierta es técnicamente superior al otro, ya que los resultados de la cirugía va depender en gran medida de la técnica y la experiencia del cirujano. Por tanto, es imperativo establecer pautas basadas en evidencia con respecto a la determinación de la competencia y la capacitación estandarizada para cirujanos con supervisión obligatoria (Liu et al., 2019). 


\section{Conclusiones}

No se presentaron problemas para la evaluación del diagnóstico, se realizaron los exámenes pertinentes y requeridos por el especialista para dar con el estado del paciente. Los datos que se evidencian en los exámenes concuerdan con el razonamiento inicial del especialista. Nuestro estudio resalta que es indispensable realizar una evaluación o valoración de cada paciente de manera integral $\mathrm{y}$, elegir el método de operación más adecuado para maximizar los beneficios del paciente. Para esto se requiere de buen entrenamiento $\mathrm{y}$ educación tanto en laparoscopia y robótica bajo supervisión de médicos con alto nivel de experiencia y en un centro hospitalario donde se hagan este tipo procedimientos de manera constante. También se debería continuar haciendo más ensayos clínicos en centros de alto volumen ya que es necesario para el desarrollo de nuevas técnicas quirúrgicas y uso de la tecnología en cirugía.

\section{Referencias}

Buanes, T. y Edwin, B. (2018). Long term oncological outcome of laparoscopic techniques in pancreatic cancer. World Journal Gastrointest Endoscopy, 10(12), 383-391.

Burgos L. (2008). Colangiocarcinoma. Actualización, Diagnostico y Terapia. Revista Medica De Chile, 136, 56-78.

Cardinale, V., Semeraro, R. y Torrice, A. (2010). Intra-hepatic and extra-hepatic cholangiocarcinoma: angiocarcinoma: new insight into epidemiology and risk factors. World Journal Gastrointestinal Oncology, 2 (11), 407-416.
Chapa-Azuela, O., Roldán-García, J., Díaz-Martínez, J., y Etchegaray-Dondé, A. (2017). Pancreatoduodenectomía totalmente laparoscópica. Primer caso reportado en México. Cirugía y Cirujanos, 84 (5), 344-349.

Kornaropoulos, M., Moris, D., Beal W, E., Makris, M. C., Mitrousias, A. y Petrou, A. (2017). Total robotic pancreaticoduodenectomy: a systematic review. Surgical Endoscopy, 31(11), 4382-4392.

Labib, P. L., Goodchild, G. y Pereira, S. P. (2019). Molecular Pathogenesis of Cholangiocarcinoma. BMC Cancer, 19, 185

Lalli, R., Merritt, N. y Schlachta, C. (2018). Robotic-assisted, spleen-preserving distal pancreatectomy for a solid pseudopapillary tumour in a pediatric patient: a case report and review of the literature. Journal of Robotic Surgery, 13(2), 325-329.

Liu, M., Ji, S., Xu, W., Liu, W., Qin, Y., Hu, Q., Sun, Q., Zhang, Z., Yu, X. y Xu, X. (2019). Laparoscopic pancreaticoduodenectomy: are the best times coming? World Journal of Surgical Oncology, 17(81), 1-11.

Mumtaz, K., Hamid , S. y Jafri, W. (2007). Endoscopic retrograde cholangiopancreaticography with or without stenting in patients with pancreaticobiliary malignancy, prior to surgery. Cochrane Database of Systematic Reviews (2), 1-8.

Peng, L., Lin, S., Li, Y. y Xiao, W. (2016). Systematic review and meta-analysis of robotic versus open pancreaticoduodenectomy. Surgical endoscopy, 31(8), 3085-3097.

Ridruejo, B. y Jimenez, S. (2006). Colangiocarcinoma infectado. Anales de Medicina Interna, 23 (7), 335-337.

Rizvi, S., Shahid A, K., Hallemeier, C. y Robin K, R. (2017). Cholangiocarcinoma-evolving concepts and therapeutic strategies. 
Nature Reviews Clinical Oncology,

15(2), 95-111.

Wang, S., Shi, N., You, L., Dai, M. y Zhao, Y. (2017)

Minimally invasive surgical approach

versus open procedure for

pancreaticoduodenectomy. Medicine, 96 (50), 1-10. 